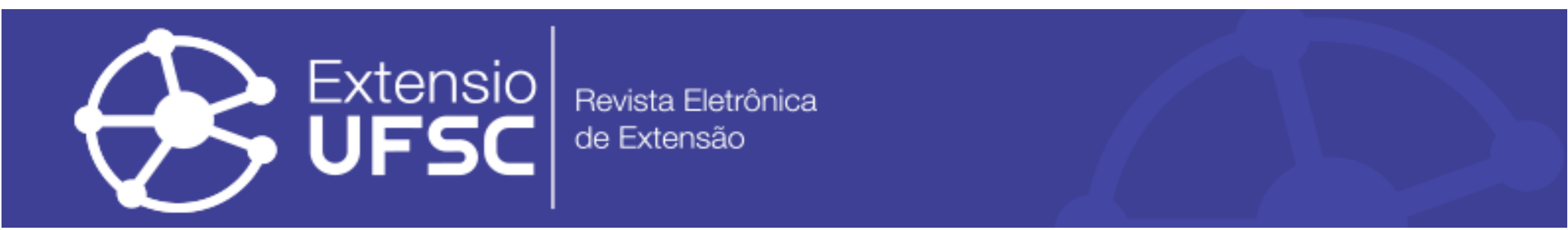

\title{
V SEMANA DO CÉREBRO DA UENP: AUTOCRÍTICA COM BASE NO FEEDBACK
}

\author{
Ohana Turcato Macacare \\ Faculdade de Medicina de Ribeirão Preto \\ ohanamacacare@gmail.com \\ Millena Lana Correia de Carvalho \\ Universidade Estadual do Norte do Paraná \\ millenalana10@gmail.com
}

\author{
Pollyanna Ferreira de Moura \\ Universidade Estadual do Norte do Paraná \\ pollyannamoura2010@gmail.com \\ Ellen Mayara Souza Cruz \\ Universidade Estadual de Londrina \\ ellensouzaq@gmail.com
}

Roberta Ekuni

Universidade Estadual do Norte do Paraná robertaekuni@uenp.edu.br

\section{Resumo}

O presente relato de extensão universitária visa apresentar a V Semana Nacional do Cérebro da UENP, focando no perfil da comunidade atingida pela ação. Essa ação ocorreu entre os dias 27 e 29 de março de 2019 e envolveu palestras sobre Neurociências com foco na saúde mental, pesquisas laboratoriais e inovações tecnológicas na área. Como meio de divulgação, utilizou-se mídias online. Os ouvintes se inscreveram via Google Forms. Como resultado, 358 pessoas se inscreveram, sendo que 256 pessoas estiveram presentes no primeiro dia, 255 no segundo e 60 no terceiro dia do evento (no bar, com lotação limitada). Apesar de ser uma ação extensionista, $90 \%$ dos ouvintes eram estudantes de graduação, mas que não necessariamente são da área de Neurociência, o que demonstra que o evento ainda está restrito ao ambiente acadêmico. Desse modo, para atingir a comunidade para além dos muros da universidade, é necessário repensar no formato desse tipo de ação extensionista, desde a divulgação, até a execução.

Palavras-chave: Neurociências. Extensão Universitária. Divulgação Neurocientífica.

\section{BRAIN AWARENESS WEEK OF UENP: SELF-CRITICISM BASED ON FEEDBACK}

\begin{abstract}
The present report of the university extension aims to present the 5th Brain Awareness Week of UENP, focusing on the community's profile affected by the action. This action took place from March 27 to 29, 2019, and involved lectures on Neurosciences with a focus on mental health, laboratory research, and technological innovations in the area. As a means of dissemination, online media were used. Listeners signed up through Google Forms. As a result, 358 people signed up, with 256 people attending the first day, 255 on the second, and 60 on the third day of the event (at the bar, with limited capacity). Despite being an extension action, $90 \%$ of the listeners were undergraduate students, but they are not necessarily from the area of Neuroscience, which shows that the event is still restricted to the academic environment. Thus, in order to reach the community beyond the walls of the university, it is necessary to rethink the format of this type of extension action, from disclosure to execution.
\end{abstract}

Keywords: Neuroscience. University Extension. Neuroscientific Dissemination.

\section{SEMANA DEL CÉREBRO DE LA UENP: AUTOCRÍTICA BASADA EN LA RETROALIMENTACIÓN}

\section{Resumen}

El presente informe de extensión universitaria tiene como objetivo presentar la V Semana Nacional del Cerebro de la UENP, centrándose en el perfil de la comunidad afectada por la acción. Esta acción tuvo lugar entre el 27 y el 29 de marzo de 2019, que involucró conferencias sobre Neurociencias con enfoque en salud mental, investigación de laboratorio e innovaciones tecnológicas en el área. Como medio de difusión se utilizaron medios online. Los oyentes se registraron a través de Google Forms. Como resultado, se inscribieron 358 personas, con 256 asistentes el primer día, 255 el segundo y 60 el tercer día del evento (en el bar, con aforo limitado). A pesar de ser una acción de extensión, el $90 \%$ de los oyentes eran estudiantes de pregrado, pero no necesariamente del área de Neurociencias, lo que demuestra que el evento aún está restringido al ámbito académico. Así, para llegar a la comunidad más allá de los muros de la universidad, es necesario repensar el formato de este tipo de acciones de extensión, desde la divulgación hasta la ejecución.

Palavras clave: Neurociencia. Extensión Universitaria. Difusión Neurocientífica. 


\section{INTRODUÇÃO}

As Neurociências, conjunto de ciências que estudam o sistema nervoso, ditam o século 21 como o século do cérebro, pois foi a partir daí que muitos estudos foram realizados sobre as funções neurais humanas (VENTURA, 2010). Paralelamente com esse desenvolvimento, surge um fenômeno conhecido como "neurofilia", ou seja, um "apetite" por informações sobre o cérebro (ABI-RACHED, 2008). Entretanto, muitos entusiastas sobre o cérebro acabam acreditando em informações equivocadas, conhecidas como neuromitos (OECD, 2002). Essas notícias falsas (fake news) são espalhadas e influenciam a tomada de decisões, muitas vezes, até mesmo quando corrigidas com informações corretas comprovadas cientificamente (LEWANDOWSKY et al., 2017).

Nesse sentido, é importante popularizar os conhecimentos neurocientíficos, para a comunidade em geral, alunos e professores de escolas particulares ou públicas (BUCK et al., 2014). Uma vez que as pessoas tenham conhecimento sobre o assunto, ele deve ser levado em consideração no momento da tomada de decisão.

Uma outra vantagem, além de popularizar as Neurociências, é que a divulgação pode despertar o interesse dos envolvidos nessa matéria e áreas semelhantes (MARTINS; MELOCARPES, 2014). Além disso, podem auxiliar na formação dos alunos de graduação envolvidos na divulgação (neuro)científica (VARGAS et al., 2014).

Uma das iniciativas que visa essa popularização é a "Semana Internacional do Cérebro" (do inglês Brain Awareness Week), uma iniciativa global de divulgação das Neurociências, promovida pela Dana Alliance for Brain Initiatives. No Brasil a "Semana Nacional do Cérebro" é chancelada, desde 2012, pela Sociedade Brasileira de Neurociência e Comportamento (SBNeC) (BUCK et al., 2014) e há registros de ações, ligadas a esta iniciativa, em todas as regiões brasileiras (BUCK et al., 2016).

A “Semana Nacional do Cérebro" ocorre no mês de março, quando, durante uma semana pré-estabelecida, iniciativas de popularizar as Neurociências são realizadas via palestras, exposições, cine debate, cursos ou pôsteres, bem como uma mistura de ações, por exemplo, via palestras e exposições (BUCK et al., 2016).

As universidades, públicas e particulares, são os principais centros organizadores da "Semana Nacional do Cérebro" (BUCK et al., 2016). Partindo da base constituinte de uma universidade, a qual se baseia em ensino, pesquisa e extensão (FORPROEX, 2012), a "Semana Nacional do Cérebro" contribui para com a Extensão Universitária, visto que a interligação do 
meio acadêmico com a população se fortalece pela extensão, que compartilha de seu conhecimento e assume o compromisso com a sociedade (FORPROEX, 2012).

O Programa de Extensão “Grupo de Estudos em Neurociência” (GEN) também participa dessa iniciativa desde 2013 (EKUNI et al., 2014), com palestras ministradas tanto por profissionais, quanto por membros do GEN, respeitando o tripé "Ensino, Pesquisa e Extensão" (MACACARE et al., 2018). Todavia, foca no caráter pedagógico da extensão universitária em conjunto com a pesquisa, de modo que amplie o tema para toda a comunidade (COELHO, 2014).

Um dos pontos importantes é que as ações extensionistas devem ir para fora da universidade, ou seja, ser ações extramuros (FERNANDES et al., 2016). O objetivo das atividades extramuros é promover um ambiente atrativo, onde a audiência em geral pode desenvolver um interesse na ciência, interagindo diretamente com os cientistas, mesmo com pouca ou nenhuma formação científica formal (ROBINSON et al., 2017).

Fazer a comunicação do estudo científico para a população proporciona inúmeros benefícios tanto para os cientistas quanto para a comunidade, visto que é uma forma de mostrar para a população a importância dos produtos da ciência para a sociedade (GONZAGA et al., 2017). Visto por outro ângulo, essa interação cientista-sociedade também pode se tornar uma iniciativa que entusiasma os pesquisadores a levarem suas descobertas para o público, participando de conversas em um ambiente descontraído, disseminando informações científicas (PAUL; MOTSKIN, 2016).

Um exemplo dessas ações extramuros é o Pint of Science (do inglês “caneca de ciência”), que acontece em ambientes não formais de ensino, ou seja, fora do contexto de sala de aula, como em bares, lanchonetes e restaurantes (FERRACIOLI et al., 2019). A execução nesses ambientes extrovertidos possibilita uma maior interação entre cientistas e o público, distanciando-se das aulas formais ou palestras acadêmicas (GONZAGA et al., 2017).

Diante disso, a "V Semana Nacional do Cérebro da Universidade Estadual do Norte do Paraná (UENP)" visou realizar palestras e mesas redondas objetivando divulgar neurociência durante três dias de evento, tanto dentro como fora da Universidade.

Vista a adesão do evento por parte do público (MACACARE et al., 2018), a V edição do evento procurou conhecer melhor o perfil de seus ouvintes, iniciativa a qual, segundo Gonzaga e colaboradores (2017), propicia melhorias para próximas edições do evento bem como traz informações úteis para futuros eventos similares. Assim, os objetivos do presente trabalho foram relatar, de forma detalhada, a organização do evento, bem como realizar uma autocrítica com base no feedback feito pelos ouvintes. 


\section{MATERIAIS E MÉTODOS}

\section{O evento}

Nos dias 27, 28 e 29 de março de 2019, ocorreu a quinta edição da "Semana Nacional do Cérebro da Universidade Estadual do Norte do Paraná”. A presente ação extensionista foi realizada no Campus Luiz Meneghel, na cidade de Bandeirantes - PR. Nos dois primeiros dias, o evento foi realizado dentro da Universidade, no auditório Thomaz Nicoletti e, no terceiro dia, foi uma ação extramuros que ocorreu em um bar situado na mesma cidade.

A iniciativa de realizar o terceiro dia do evento em um bar ocorreu pela primeira vez durante a IV edição do evento, realizada no ano de 2018 (CESÁRIO et al., 2019). Assim, por meio de relatos positivos dos ouvintes, a ação se repetiu durante a V edição. A organização e execução dessa ação extensionista foi realizada por meio do Programa de Extensão "Grupo de Estudos em Neurociência” (GEN) (EKUNI et al., 2014).

Os membros do GEN debateram sobre possíveis temas e palestrantes nas reuniões semanais e, levando em consideração a logística (palestrantes não deveriam cobrar pela palestra e de preferência morar perto da cidade), chegaram nas seguintes temáticas: "Emoções e Expressões Faciais", "Neurobiologia do Abuso de Drogas", "Máquinas que aprendem: será que as máquinas serão capazes de simular um cérebro humano?” e "Saúde Física na Construção da Saúde Mental”. Cada palestra deveria ser programada para durar até cinquenta minutos e deveriam ser organizadas de tal forma que houvesse uma interação entre os palestrantes e os ouvintes.

\section{Divulgação do evento e inscrições do público}

Para divulgar o evento para o público-alvo (docentes, discentes, alunos de colégios públicos e particulares da cidade e região e a comunidade em geral), foi criada uma página de evento no Facebook, na qual todas as informações referentes ao evento foram publicadas, como cronograma, informação dos palestrantes e divulgação do coffee solidário, estilo ao realizado anteriormente (MACACARE et al., 2018).

Devido à escassez de recurso financeiro, o coffee solidário representa uma alternativa para realizar um evento gratuito, pois os ouvintes são convidados a levar qualquer alimento, se assim desejassem. Os alimentos ficam disponíveis em uma mesa para que todos se sirvam, sendo este um momento de confraternização. 
Para fins de divulgação também houve a confecção de um flyer virtual para ser compartilhado em outras redes sociais, contendo todas as informações necessárias como o dia, horário, local e palestras. O mesmo foi distribuído via Whatsapp e Instagram.

O evento foi gratuito e a inscrição do mesmo foi realizada por uma plataforma online chamada Google Forms. Ao realizar a inscrição, os ouvintes eram convidados a responder um questionário com informações demográficas para se ter mais informações sobre o público-alvo.

Outras perguntas não-obrigatórias estavam no formulário: i) “Assinale a opção a qual corresponde com sua escolaridade" (as opções eram desde a realização do Ensino Fundamental até o Doutorado); ii) "Se estudante de curso superior, qual curso e instituição"; iii) "Se possui curso superior completo, qual curso e instituição" iv) "Como soube do evento" (opções: soube do evento por meio de divulgação online, por indicação de um colega ou de outra forma); v) "Já participou de outras edições do evento? Se sim, de 0 a 10, qual nota dá para o evento" e vi) "Caso seja do seu interesse, este campo está aberto para sugestões, caso não seja clique em finalizar".

Após o evento, foi divulgado um outro questionário pelo Facebook convidando os ouvintes a responderem. Destaca-se que nenhum dos questionários era obrigatório e apenas teve a finalidade de a equipe executora saber mais informações sobre o público que se interessa pelo evento.

No questionário pós-evento, as perguntas visaram inquerir a opinião do ouvinte em relação ao evento: i) "Se estudante Universitário, qual curso?”; ii) "Numa escala de 1 a 10 (onde 1 representa "Não Gostei Nenhum Pouco" e 10 representa "Gostei Muito") quanto você gostou da V edição do nosso evento?”, iii) "Brevemente, nos diga qual é sua opinião sobre as palestras que você assistiu."; iv) "Se você participou de outras edições do evento, em uma escala de 1 a 10 (onde 1 representa "Não Melhorou em Nada" e 10 "Melhorou Muito"), qual a sua opinião sobre a V edição do evento."; v) "Se você participou do último dia do evento, realizado em um bar, em uma escala de 1 a 10 (onde 1 representa "Não Gostei Nada da Ideia" e 10 "Gostei Muito da Ideia") o quando você gostou?”; vi) “Quais os temas que você gostaria que fosse falado nas próximas edições do evento?" e vii) "Muito obrigada por responder este questionário. Use este espaço para deixar elogios, críticas e sugestões caso quiser".

\section{Primeiro Dia do Evento}

O primeiro dia do evento foi marcado por abertura cultural realizada por uma Bateria Universitária, sendo esta formada por alunos de diversos cursos da UENP. A bateria foi escolhida para realizar a abertura, principalmente por ter como base estruturante a integração dos alunos em suas ações, bem como forma de divulgar as ações culturais realizadas dentro da Universidade. 
Após a abertura cultural deu-se início a palestra da noite, intitulada "Emoções e Expressões Faciais". Tal palestra foi apresentada por duas discentes de Ciências Biológicas integrantes do GEN. A iniciativa de membros do grupo de estudos palestrarem vem sendo realizada desde as últimas edições do evento. Na palestra foi abordado os seguintes temas: i) "as diferenças entre humor e estado de ânimo" (EKMAN, 2011); ii) "Por que e quando nos emocionamos" seguindo as concepções Darwinianas (EKMAN, 2003) e iii) “expressões emocionais" (EKMAN, 2011).

Após a palestra, foi apresentado um episódio da série "Lie To Me" visando a possibilidade de associar o conteúdo ministrado na palestra com as cenas do episódio. Divulgar neurociência por meio de filmes, estilo Neuromovies, pode ser uma estratégia útil para auxiliar no aprendizado (MARIQUITO; OTOMURA; EKUNI, 2020) e é uma pratica recorrente nas edições dos eventos anteriores (MACACARE et al., 2018; CESÁRIO et al., 2019).

\section{Segundo Dia do Evento}

O segundo dia de evento iniciou-se com a palestra "Neurobiologia do Abuso de Drogas", ministrada por duas palestrantes, Doutorandas pela Universidade de São Paulo - campus Ribeirão Preto). As palestrantes abordaram sobre: i) "principais as características sobre as drogas de abuso" (BRANDÃO; GRAEFF, 2014); ii) "o condicionamento Pavloviano e o efeito das drogas de abuso" (SIEGEL, 2005); iii) “a circuitaria límbica e drogas de abuso” (KANDEL et al., 2014); e iv) "a memória relacionada ao contexto e sua associação ao abuso de drogas" (WEISS, 2005; PALIARIN; INCROCCI; NOBRE, 2018). A interlocução entre os ouvintes e as palestrantes foi mantida o tempo todo e estes tinham a liberdade de realizar perguntas durante a palestra.

Em seguida, ocorreu a apresentação da palestra "Máquinas que aprendem: será que as máquinas serão capazes de simular um cérebro humano?”, a qual foi apresentada por um professor assistente da UENP, doutorando em informática na PUC-PR).

O palestrante abordou a definição de inteligência artificial, suas aplicações no dia a dia e projetos existentes, focando nas seguintes questões: i) "Como humanos e as máquinas aprendem”, comparando-os; ii) "O que a inteligência artificial já realiza" (e.g. BARRETO, 2002); e iii) "Será que as máquinas são capazes de simular um cérebro humano?”. Foi aberto espaço para perguntas ao final da palestra, no qual os ouvintes mostraram-se interessados e curiosos sobre a temática.

\section{Terceiro Dia do Evento}

O terceiro dia do evento ocorreu em um bar, estilo "Pint of science"). A palestra foi ministrada por uma psicóloga com ênfase em clínica comportamental e mestranda em Análise do 
Comportamento pela Universidade Estadual de Londrina, abordando o tema "Saúde Física na Construção da Saúde Mental”.

A palestra focou na: i) "Integração da saúde mental com a saúde física” mostrando que essa integração pode ajudar na prevenção e tratamento de transtornos psíquicos, dessa forma melhorando a qualidade de vida das pessoas (PELUSO; ANDRADE, 2005). No senso comum, mente e corpo são separados, entretanto, eles são integrados (JUNIOR, 2008); ii) “importância da assistência multifuncional", com o foco de não procurar somente psicólogo ou somente ortopedista, mas cuidar da saúde como um todo para a integração desses sistemas mente e corpo; iii) "prevenção para doenças" por meio de redes de apoio como amigos, familiares, acesso a saúde de modo que ajude a tratar os transtornos psíquicos.

Ao final da palestra, e também durante esta, o público pôde realizar questionamentos.

\section{RESULTADOS E ANÁLISES}

$\mathrm{Na}$ inscrição on-line, 358 pessoas se inscreveram e, destas, 78\% aceitaram responder o questionário. Esses tinham idade média de 20,61 anos (DP $\pm 3,19$ ), e 78\% eram do sexo feminino. Participaram, efetivamente, no primeiro e segundo dia, respectivamente, 256 e 220 pessoas.

No segundo dia, além daqueles que se inscreveram por meio da plataforma online, houve a participação de 35 alunos de uma escola pública de um município próximo à universidade, estudantes do Ensino Médio e da Educação de Jovens e Adultos. A participação desses alunos foi possível pois uma professora viu a divulgação do evento nas redes sociais e demonstrou interesse em participar, o que evidencia a importância das redes sociais para a ampla divulgação destes eventos. Os mesmos relataram que só puderam participar no segundo dia por causa da disponibilidade de transporte.

Por fim, no terceiro dia do evento, realizado em um bar com lotação limitada, participaram 60 pessoas.

Em relação à escolaridade, $90 \%$ dos ouvintes são estudantes de graduação (Tabela 1). Entre os estudantes de graduação, há uma maior participação de alunos da área da saúde, 41\% deles são estudantes de Ciências Biológicas, 30\% de Enfermagem e 16\% de Medicina Veterinária (Tabela 2).

A principal forma de divulgação do evento ocorreu pelo compartilhamento onine de folders informativos, sendo que $62 \%$ dos ouvintes relataram tomar conhecimento do evento por meio de divulgação on-ine. Assim como nos anos anteriores, também houve a participação da comunidade 
na divulgação do evento, visto que $36 \%$ dos ouvintes souberam do evento através de um colega (Tabela 3).

Do total de inscritos no evento que responderam às perguntas, $51 \%$ já tinham participado de outras edições. Em relação à nota, numa escala de 0 a 10, que eles davam para as outras edições do evento, a média foi de 9,17 (DP $\pm 1,16$ ). Como feedback, os organizadores receberam mensagens parabenizando o evento, a iniciativa e ressaltando a importância que eventos de extensão têm para a universidade. Uma mensagem interessante foi a ideia dos alunos de criar uma parceria entre o evento e cursos da área da saúde.

\begin{tabular}{|c|c|}
\hline Escolaridade & $\mathbf{0}$ \\
\hline Estudante de Graduação & $90 \%$ \\
\hline Ensino Médio Completo & $5 \%$ \\
\hline Curso superior completo & $3 \%$ \\
\hline Especialização ou mestrado & $1 \%$ \\
\hline
\end{tabular}

Quadro 1 - Escolaridade, em porcentagem, dos ouvintes. Fonte: elaborado pelas autoras

\begin{tabular}{|c|c|}
\hline Curso de Graduação & $\mathbf{\%}$ \\
\hline Ciências Biológicas & $41 \%$ \\
\hline Enfermagem & $30 \%$ \\
\hline Medicina Veterinária & $16 \%$ \\
\hline Ciências da Computação & $9 \%$ \\
\hline Letras & $2 \%$ \\
\hline Pedagogia & $1 \%$ \\
\hline Agronomia & $1 \%$ \\
\hline Outros & $1 \%$ \\
\hline
\end{tabular}

Quadro 2 - Relação, em Porcentagem, dos Cursos de Graduação Realizados Pelos Ouvintes. Fonte: elaborado pelas autoras

\begin{tabular}{|c|c|}
\hline Como Soube do Evento & $\mathbf{\%}$ \\
\hline Divulgação Online & $62 \%$ \\
\hline Por Um Colega & $36 \%$ \\
\hline Outros & $3 \%$ \\
\hline
\end{tabular}

Quadro 3 - Relação, em Porcentagem, Sobre Como os Ouvintes Souberam do Evento. Fonte: elaborado pelas autoras

Em relação às palestras, perguntas foram feitas ao final de todas pelos ouvintes e devidamente respondidas pelos palestrantes, com base em seus conhecimentos prévios. Alguns ouvintes abordaram os palestrantes após o encerramento para realizar perguntas que por algum motivo não realizaram durante as palestras. 
Não houve muita adesão no questionário pós-evento. Apenas 29 pessoas responderam, destas 93\% era do público feminino com a idade média de 20,24 (DP $\pm 2,18$ ). Todos que responderam eram estudantes universitários dos cursos de Ciências Biológicas (66\%), Enfermagem (31\%) e Medicina Veterinária (3\%). Em relação a quanto os ouvintes gostaram da V edição do evento, obteve-se uma média de 7,31 (DP $\pm 2,97)$. No que diz respeito à opinião dos ouvintes que participaram do terceiro dia do evento, quanto à ideia do último dia ser em um bar, a média da nota obtida foi de 7,87 (DP $\pm 3,00)$.

Os ouvintes que participaram de outras edições julgaram que a $\mathrm{V}$ edição do evento teve a nota de 6,06 (DP $\pm 3,30)$ melhor em relação às outras.

Os resultados mais interessantes em relação ao questionário pós-evento vêm dos espaços onde os ouvintes tinham liberdade para dizer suas opiniões, sobre alguns tópicos, com suas próprias palavras. No tópico "Brevemente, nos diga qual é sua opinião sobre as palestras que você assistiu", comentários elogiando as palestras foram feitos, além de comentários sobre a relevância dos assuntos abordados, os quais os ouvintes julgaram apresentar utilidade na vida acadêmica e não acadêmica.

Ainda, foi comentado, elogiosamente, sobre o fato de duas alunas palestrarem no evento. Tal tópico, porém, também apresentou críticas, como, por exemplo, terem julgado o evento como desorganizado e que as palestrantes eram despreparadas para palestrar. Ou seja, comentários tanto elogiosos quanto críticas foram feitas.

O tópico "Quais os temas que você gostaria que fosse falado nas próximas edições do evento?" foi pensado para nortear os organizadores do evento quanto à expectativa do público sobre os temas a serem abordados nas próximas edições. Como resposta, foram sugeridas: "emoções"; "como lidar com frustrações do cotidiano"; "planejamento de metas"; "saúde mental”; "as reações do cérebro com o medo"; e "Alzheimer".

No último tópico "Use este espaço para deixar elogios, críticas e sugestões caso quiser", houve comentários positivos ao evento. Um comentário que se destacou foi o fato de o evento ter sido organizado majoritariamente por mulheres universitárias e que elas estão cada vez mais ocupando seu espaço. O único comentário negativo foi em relação ao último dia do evento ser realizado em um bar. O ouvinte relatou que achava que tal iniciativa era imprópria e que considera uma "desculpa” para estar dentro de um bar e que este não é o papel da universidade.

Este comentário negativo, que diz respeito à realização do último dia do evento em um bar, vai de encontro à proposta de sucesso de popularização científica como o Pint of Science, que busca nos espaços não formais um meio de se mostrar os produtos da ciência bem como realizar um 
diálogo direto com o público (PONTES, 2019), o que pode sugerir um pensamento conservador tendencioso por parte do ouvinte que não condiz com a proposta do evento.

O fato de $90 \%$ dos inscritos no evento serem alunos de graduação demonstra que o presente evento de extensão, neste formato, está restrito ao ambiente acadêmico, assim como a Semana do Cérebro realizada em Uruguaiana, no estado do Rio Grande do Sul (MARTINS; MELLO-CARPES, 2014). Além dos universitários, também foi alcançado, em menor escala, a comunidade do norte pioneiro do Paraná. Entretanto, ainda que a maioria dos participantes sejam acadêmicos da UENP, o mesmo ainda cumpre o seu papel de divulgação neurocientífica, visto que há poucos projetos na instituição abordando esse tópico.

Em relação ao feedback, a sugestão de fazer evento na área da saúde em parceria com o GEN reforça o perfil do público em ser majoritariamente da área da saúde. Nota-se que os ouvintes que responderam os questionários em sua maioria eram do curso de Ciências Biológicas (66\%), talvez pelo fato da coordenadora do evento estar vinculada a esse colegiado.

O fato de metade dos ouvintes já terem participado anteriormente pode demostrar que o evento apresenta uma boa qualidade, o que pode ser demonstrado pela nota que os ouvintes deram para os eventos anteriores. Essa nota corrobora os relatos positivos feitos pelos ouvintes da edição passada para os organizadores (MACACARE et al., 2018). No questionário pós-evento deve-se levar em consideração a baixa adesão do público, o que pode ser refletido na nota. No geral, os ouvintes deram uma nota boa para o evento.

Outro ponto interessante é que não somente os ouvintes percebem a importância de acadêmicos palestrarem, prática que vem sendo adotada pelo evento, mas também os mesmos veem essa prática como sendo de suma importância para a formação acadêmica (MACACARE et al., 2018). Isso reforça o papel pedagógico da extensão universitária (COELHO, 2014).

Por fim, destaca-se o comentário referente ao posicionamento quanto às questões de gênero por parte da comissão organizadora, majoritariamente feminina. Realizar a divulgação científica destacando o papel da mulher pesquisadora/aluna traz contribuições positivas no incentivo aos ouvintes a também ingressarem neste meio (MELLO-CARPES; LLORET, 2018). Dado a importância e iniciativas atuais de se incentivar mulheres na ciência, esse comentário é relevante. 


\section{CONSIDERAÇÕES FINAIS}

Os resultados da análise da comunidade alcançada mostram que a divulgação online é eficiente, entretanto, eventos como esse ainda atingem majoritariamente o meio acadêmico. No entanto, vale ressaltar que mesmo sendo acadêmicos, não significa que são da área de Neurociências, o que mostra que a ação ainda cumpre o papel de divulgação científica. Visando atingir outro público, é necessário repensar estratégias e atratividades, para aumentar a proporção de ouvintes de fora da universidade. Analisar a opinião dos ouvintes é de suma importância pois o feedback do público garante que as informações sejam passadas de maneira clara e proveitosa, para que os próximos eventos tenham melhorias (GONZAGA; SILVEIRA; LANNES, 2017), e também para servir como base para outros grupos realizarem suas ações. Ademais, o fato de abrir possibilidade para acadêmicos palestrarem, auxilia na formação dos mesmos, evidenciando o papel pedagógico da extensão universitária.

\section{AGRADECIMENTOS}

Ao apoio da Direção do Campus Luiz Meneghel e da Pró-Reitoria de Extensão e Cultura da UENP. Aos organizadores e monitores da "V Semana o Cérebro da UENP". Agradecimento especial aos alunos do GEN que auxiliaram a coordenadora na organização.

\section{REFERÊNCIAS}

ABI-RACHED, J. M. The implications of the new brain sciences. EMBO reports, v. 9, n. 12, p. 1158-1162, 2008.

BARRETO, J. M. Introdução às redes neurais artificiais. Florianópolis: Ufsc, 2002.

BRANDÃO, M. L.; GRAEFF, F. G. Neurobiologia dos transtornos mentais. São Paulo: Editora Atheneu, 2014.

BUCK, C. H. et al. II Semana Nacional do Cérebro (ISNC/2013): popularizando as neurociências. Neurociências, v. 10, n. 2, p. 73-84, 2014.

BUCK, C. H. et al. Semana Nacional do Cérebro 2014: grandes eventos em foco. Neurociências •, v. 12, n. 1, p. 4-20, 2016. 
CESÁRIO, R. C. et al. IV SEMANA DO CÉREBRO: A Neurociência Vai A Universidade E Ao Bar. CATAVENTOS-Revista de Extensão da Universidade de Cruz Alta, v. 11, n. 2, p. 11 24, 2019.

COELHO, G. C. O papel pedagógico da Extensão Universitária. Em Extensão, v. 13, n. 2, p. 11 24, 2014.

EKMAN, P. A linguagem das emoções. São Paulo: Lua de Papel, 2011.

EKMAN, P. Darwin, deception, and facial expression. Annals of the New York Academy of Sciences, v. 1000, n. 1, p. 205-221, 2003.

EKUNI, R. et al. Projeto de Extensão "Grupo de Estudos em Neurociência": divulgando neurociência e despertando vocações. Revista Brasileira de Extensão Universitária, v.5, n. 2, p. 55-59, 2014.

FERNANDES, T. et al. A Extensão Universitária Como Elo Fundamental Para Troca De Saberes E Resgate Cultural Em Espaços Não-Formais. Raízes E Rumos, v. 4, n. 1, p. 3, 2016.

FERRACIOLI, L. et al. Festival Pint of Science e Inclusão Cultural: A Ciência na Conversa de Bar. Revista Guará, v. 6, n. 10, 2019.

FÓRUM DE PRÓ-REITORES DE EXTENSÃO DAS UNIVERSIDADES PÚBLICAS BRASILEIRAS. FORPROEX. Política Nacional de Extensão Universitária. Manaus: [s.n.]. Disponível em: https://www.ufmg.br/proex/renex/images/documentos/2012-0713PoliticaNacional-de-Extensao.pdf. Acesso em: 10 nov. 2019.

GONZAGA, L. L.; SILVEIRA, J. R. A.; LANNES, D. Ciência fora dos muros da universidade: o caso do Pint of science na cidade do Rio de Janeiro, Brasil. Ciência e Cultura, v. 69, n. 3, p. 5659, 2017.

JUNIOR, C. A. P. Sobre a importância do corpo para a continuidade do ser. Revista Mal-Estar e Subjetividade, v. 8, n. 4, p. 927-958, 2008.

KANDEL et al. Princípios de Neurociências. 5. ed. AMGH Editora, 2014. p. 938-969.

LEWANDOWSKY, S.; ECKER, U. K. H.; COOK, J. Beyond Misinformation: Understanding and Coping with the "Post-Truth" Era. Journal of Applied Research in Memory and Cognition, v. 6, n. 4, p. 353-369, 2017.

MACACARE, O. T. et al. Semana do cérebro: divulgando a neurociência e integrando ensino, pesquisa e extensão. Expressa Extensão, v. 23, n. 3, p. 52-65. 2018.

MARIQUITO, M. T.; OTOMURA, F. H.; EKUNI, R. NeuroMovies: ensinando neurociência pelas lentes dos filmes. Atos de Pesquisa em Educação (FURB), 2019 - no prelo.

MARTINS, A.; MELLO-CARPES, P. B. A proposal for undergraduate students' inclusion in brain awareness week: promoting interest in curricular neuroscience components. Journal of Undergraduate Neuroscience Education, v. 13, n. 1, p. A41, 2014. 
MELLO-CARPES, P. B.; LLORET, A. Women in (neuro) science: report of a meeting held at the University of Valencia, Spain, in February 2018. Advances in Physiology Education, v. 42, p. 668-671. 2018.

NOGUEIRA, M. D. P. O Fórum de Pró-reitores de Extensão das Universidades Públicas Brasileiras: um ator social em construção. Interfaces-Revista de Extensão da UFMG, v. 1, n. 1, p. 35-47, 2013.

OECD - Organization for Economic Cooperation and Development. 2002. Learning Seen from a Neuroscientific Approach. OECD Publications Service, Paris. http://browse. oecdbookshop.org/oecd/pdfs/product/9102021e.pdf

PALIARIN, F.; INCROCCI, R. M; NOBRE, M. J. Behavioral and Auditory Electrophysiological Rebound as a Compensatory Response to the Reinforcing Effects of Morphine. Neuroscience, v. 392, p. 66-76, 2018.

PAUL, P.; MOTSKIN M. Engaging the public with your research. Trends in immunology, v. 37, n. 4, p. 268-271, 2016.

PELUSO, M. A. M.; ANDRADE, L. H. S. G. Atividade física e saúde mental: a associação entre exercício e humor. Clinics, v. 60, n. 1, p. 61-70, 2005.

PONTES, N. Science in new spaces. Brazilian Dental Science, v. 22, n. 3, p. 295-296, 2019.

ROBINSON, M. T. et al. The first pint of science festival in Asia. Science Communication, v. 39, n. 6, p. 810-820, 2017.

SIEGEL, S. Drug tolerance, drug addiction, and drug anticipation. Current Directions in psychological science, v. 14, n. 6, p. 296-300, 2005.

$\begin{array}{lrrcrr}\text { SOCIEDADE } & \text { BRASILEIRA } & \text { DE } & \text { NEUROCIENCIAS } & \text { E } & \text { COMPORTAMENTO } \\ \text { SBNEC. Semana } & \text { do } & & \text { Cérebro. } & \text { Disponível } & \text { em: }\end{array}$ http://www.sbnec.org.br/site/texto.php?id_texto=9. Acesso em: 10 nov. 2019.

VARGAS, L. S. et al. Conhecendo o sistema nervoso: ações de divulgação e popularização da neurociência junto a estudantes da rede pública de educação básica. Ciências \& Cognição, v. 19, n. 2, p. 233-241, 2014.

VENTURA, D. F. Um retrato da área de neurociência e comportamento no Brasil. Psicologia: teoria e pesquisa, p. 123-130, 2010.

WEISS, F. Neurobiology of craving, conditioned reward and relapse. Current opinion in pharmacology, v. 5, n. 1, p. 9-19, 2005.

Recebido em: 27/09/2020

Aceito em: 16/08/2021 